\title{
Improved and Extended Locating Functionality on Compressed Suffix Arrays *
}

\author{
Simon Gog $^{1}$ and Gonzalo Navarro ${ }^{2}$ \\ 1 Department of Computing and Information Systems, The University of Melbourne. \\ simon.gog@unimelb.edu.au \\ 2 Department of Computer Science, University of Chile. gnavarro@dcc.uchile.cl
}

\begin{abstract}
Compressed Suffix Arrays (CSAs) offer the same functionality as classical suffix arrays (SAs), and more, within space close to that of the compressed text, and in addition they can reproduce any text fragment. Furthermore, their pattern search times are comparable to those of SAs. This combination has made CSAs extremely successful substitutes for SAs on space-demanding applications. Their weakest point is that they are orders of magnitude slower when reporting the precise positions of pattern occurrences. SAs have other well-known shortcomings, inherited by CSAs, such as retrieving those positions in arbitrary order. In this paper we present new techniques that, on one hand, improve the current space/time tradeoffs for locating pattern occurrences on CSAs, and on the other, efficiently support extended pattern locating functionalities, such as reporting occurrences in text order or limiting the occurrences to within a text window. Our experimental results display considerable savings with respect to the baseline techniques.
\end{abstract}

\section{Introduction}

Suffix arrays [12121 are text indexing data structures that support various pattern matching functionalities. Built on a text $T[1, n]$ over an alphabet $[1, \sigma]$, the most basic functionality provided by a suffix array (SA) is to count the number of times a given pattern $P[1, m]$ appears in $T$. This can be done in $O(m \log n)$ and even $O(m+\log n)$ time [21. Once counted, SAs can report each of the occ positions of $P$ in $T$ in $O(1)$ time. A suffix array uses $O(n \log n)$ bits of space and can be built in $O(n)$ time [19]18]17.

The space usage of suffix arrays, albeit "linear" in classical terms, is asymptotically larger than the $n \lg \sigma$ bits needed to represent $T$ itself. Since the year 2000, two families of compressed suffix arrays (CSAs) emerged [25]. One family, simply called CSAs $[14[15[30|31| 13$, built on the compressibility of a so-called $\Psi$ function (see details in the next section), and simulated the basic SA procedure for pattern searching, achieving the same $O(m \log n)$ counting time of basic SAs. A second family, called FM-indexes [5|6|7|1, built on the BurrowsWheeler transform [3] of $T$ and on a new concept called backward-search, which

\footnotetext{
* Funded by Fondecyt grant 1-140796, Chile and ARC grant DP110101743, Australia.
} 
allowed $O(m \log \sigma)$ and even $O(m)$ time for counting occurrences. The counting times of all CSAs are comparable to those of SAs in practical terms as well 4 . Their space usage can be made asymptotically equal to that of the compressed text under the $k$-th order empirical entropy model, and in all cases it is below $n \lg \sigma+o(n \lg \sigma)$ bits. Within this space, CSAs support even stronger functionalities than SAs. In particular, they can reconstruct any text segment $T[l, r]$, as well as to compute "inverse" suffix array entries (again, details in the next section), efficiently. Reproducing any text segment allows CSAs to replace $T$, further reducing space.

The weakest part of CSAs in general is that they are much slower than SAs at retrieving the occ positions where $P$ occurs in $T$. SAs require basically occ contiguous memory accesses. Instead, both CSA families use a sampling parameter $s$ that induces an extra space of $O((n / s) \log n)$ bits (and therefore $s$ is typically chosen to be $\Omega(\log n))$; then $\Psi$-based CSAs require $O(s)$ time per reported position and FM-indexes require $O(s \log \sigma)$. In practical terms, all CSAs are orders of magnitude slower than SAs when reporting occurrence positions 4], even when the distribution of the queries is known [8]. Text extraction complexities for windows $T[l, r]$ are also affected by $s$, but to a lesser degree, $O(s+r-l)$.

Although widely acknowledged as a powerful and flexible tool for text searching activities, the SA has some drawbacks that can be problematic in certain applications. The simplest one is that it retrieves the occurrence positions of $P$ in an order that is not left-to-right in the text. This complicates displaying the occurrences in order (unless one obtains and sorts them all), as for example when displaying the occurrences progressively in a document viewer. A related one is that there is no efficient way to retrieve only the occurrences of $P$ that are within a window of $T$ unless one uses $\Omega(n \log n)$ bits of space [20|2 26]16]. This is useful, for example, to display occurrences only within some documents of a collection ( $T$ being the concatenation of the documents), only recent news in a collection of news documents, etc.

In this paper we present new techniques that speed up the basic pattern locating functionalities of CSAs, and also efficiently support extended functionalities. Our experimental results show that the new solutions outperform the baseline solutions by a wide margin, in some cases.

1. We unify the samplings for pattern locating and for displaying text substrings into a single data structure, by using the fact that they are essentially the inverse permutations of each other. This yields improved space/time tradeoffs for locating pattern positions and displaying text substrings, especially in memory-reduced scenarios where large values of $s$ must be used.

2. The occ positions of $P$ have variable locating cost on a CSA. We use a data structure that takes $2 n+o(n)$ additional bits to report the occurrences of $P$ from cheapest to most expensive, thereby making reporting considerably faster when only some occurrences must be displayed (as in search engine interfaces, or when one displays results progressively and can show a few and process the rest in the background). Our experiments show that, when reporting less than around $15 \%$ of the occurrences, this technique is faster 
than reporting random occurrences, even when the baseline uses those extra $2 n+o(n)$ bits to reduce $s$. A simple alternative that turns out to be very competitive is just to report first the occurrences that are sampled in the CSA, and thus can be reported at basically no cost.

3. Variants of the previous idea have been used for document listing [24] and for reporting positions in text order [26. We study this latter application in practice. While for showing all the occurrences in order it is better to extract and partially sort them, one might need to show only the first occurrences, or might have to show the occurrences progressively. Our implementation becomes faster than the baseline when we report a fraction below $25 \%$ of the occurrences. The improvement increases linearly, reaching for example three times faster when reporting $5 \%$ of the occurrences. Again, we let the baseline spend those $2 n+o(n)$ extra bits on a denser sampling.

4. Finally, we extend this second idea to report the text positions that are within a given text window. While the result is not competitive for windows located at random positions of $T$, our method is faster than the baseline of filtering the text positions by brute force when the window is within the first $15 \%$ of $T$. This is particularly useful in versioned collections or news archives, when the latest versions/dates are those most frequently queried.

The improved sampling we proposed is available in this branch of SDSL: https://github.com/simongog/sdsl-lite/tree/better_sampling.

\section{Compressed Suffix Arrays}

Let $T[0, n-1]$ be a text over alphabet $[0, \sigma-1]$. Then a substring $T[i, n-1]$ is called a suffix of $T$, and is identified with position $i$. A suffix array $S A[0, n-1]$ is a permutation of $[0, n-1]$ containing the positions of the $n$ suffixes of $T$ in increasing lexicographic order (thus the suffix array uses at least $n \lg n$ bits). Since the positions of the occurrences of $P[0, m-1]$ in $T$ are precisely the suffixes of $T$ that start with $P$, and those form a lexicographic range, counting the number of occurrences of $P$ in $T$ is done via two binary searches using $S A$ and $T$, within $O(m \log n)$ time. Once we find that $S A[s p, e p]$ contain all the occurrences of $P$ in $T$, their number is occ $=e p-s p+1$ and their positions are $S A[s p], S A[s p+1], \ldots, S A[e p]$. With some further structures adding up to $O(n \log n)$ bits, suffix arrays can do the counting in $O(m+\log n)$ time 21. This can be reduced to $O(m)$ by resorting to suffix trees [34, which still use $O(n \log n)$ bits but too much space in practice.

Our interest in this paper is precisely using less space while retaining the SA functionality. A compressed suffix array (CSA) is a data structure that emulates the SA while using $O(n \log \sigma)$ bits of space, and usually less on compressible texts 25]. One family of CSAs [14 15/30]31 13] builds on the so-called $\Psi$ function: $\Psi(i)=S A^{-1}[S A[i]+1]$, where $S A^{-1}$ is the inverse permutation of the suffix array (given a text position $j, S A^{-1}[j]$ tells where in the suffix array is the pointer to the suffix $T[j, n-1])$. Thus, if $S A[i]=j, \Psi(i)$ tells where is $j+1$ mentioned 
in $S A, S A[\Psi(i)]=S A[i]+1=j+1$. It turns out that array $\Psi$ is compressible up to the $k$-th order empirical entropy of $T$ 22. With small additional data structures, $\Psi$-based CSAs find the range $[s p, e p]$ for $P[0, m-1]$ in $O(m \log n)$ time.

A second family, FM-indexes [56771, build on the Burrows-Wheeler transform $\left[3\right.$ of $T$, denoted $T^{b w t}$, which is a reversible permutation of the symbols in $T$ that turns out to be easier to compress. With light extra structures on top of $T^{b w t}$, one can implement a function called $L F(i)=S A^{-1}[S A[i]-1]$, the inverse of $\Psi$, in time at most $O(\log \sigma)$. An extension to the $L F$ function is used to implement a so-called backward-search, which allows finding the interval $[s p, e p]$ corresponding to a pattern $P[0, m-1]$ in $O(m \log \sigma)$ and even $O(m)$ time [1].

Once the range $S A[s p, e p]$ is found (and hence the counting problem is solved), locating the occurrences of $P$ requires finding out the values of $S A[k]$ for $k \in[s p, e p]$, which are not directly stored in CSAs. All the practical CSAs use essentially the same solution for locating [25]. Text $T$ is sampled at regular intervals of length $s$, and we store those sampled text positions in a sampled suffix array $S A_{s}[0, n / s]$, in suffix array order. More precisely, we mark in a bitmap $B[0, n-1]$ the positions $S A^{-1}[s \cdot j]$, for all $j$, with a 1 , and the rest are 0 s. Now we traverse $B$ left to right, and append the value $S A[i] / s$ to $S A_{s}$ for each $i$ such that $B[i]=1$. Array $S A_{s}$ requires $(n / s) \lg (n / s)+O(n / s)$ bits of space, and $B$ can be implemented in compressed form using $(n / s) \lg s+O(n / s)+o(n)$ bits 2827, for a total of $(n / s) \lg n+O(n / s)+o(n)$ bits.

To compute $S A[i]$ at search time, we proceed as follows on a $\Psi$-based CSA. If $B[i]=1$, then the position is sampled and we know its value is in $S A_{s}$, precisely at position $\operatorname{rank}_{1}(B, i)$, which counts the number of $1 \mathrm{~s}$ in $B[1, i]$ (this function is implemented in constant time in the compressed representation of $B$ [28]). Otherwise, we test $B[\Psi(i)], B\left[\Psi^{2}(i)\right]$, and so on until we find $B\left[\Psi^{k}(i)\right]=B\left[i^{\prime}\right]=$ 1. Then we find the corresponding value at $S A_{s}$; the final answer is $S A[i]=$ $S A_{s}\left[\operatorname{rank}_{1}\left(B, i^{\prime}\right)\right] \cdot s-k$. The procedure is analogous on an FM-index, using function $L F$, which traverses $T$ backwards instead of forwards. The sampling guarantees that we need to perform at most $s$ steps before obtaining $S A[i]$.

To display $T[l, r]$ we use the same sampling positions $s \cdot j$, and store a sampled inverse suffix array $S A_{s}^{-1}[1, n / s]$ with the suffix array positions that point to the sampled text positions, in text order. More precisely, we store $S A_{s}^{-1}[j]=$ $S A^{-1}[j \cdot s]$ for all $j$. This requires other $(n / s) \lg s+O(n / s)$ bits of space. Then, in order to display $T[l, r]$ with a $\Psi$-based CSA, we start displaying slightly earlier, at text position $\lfloor l / s\rfloor \cdot s$, which is pointed from position $i=S A_{s}^{-1}[\lfloor l / s\rfloor]$ in $S A$. The first letter of a suffix $S A[i]$ is easily obtained on all CSAs if $i$ is known. Therefore, displaying is done by listing the first letter of suffixes pointed from $S A[i], S A[\Psi(i)], S A\left[\Psi^{2}(i)\right], \ldots$ until covering the window $T[l, r]$. The process is analogous on FM-indexes. In total, we need at most $s+r-l$ steps.

This mechanism is useful as well to compute any $S A^{-1}[j]$ value. If $j$ is a multiple of $s$ then the answer is at $S A_{s}^{-1}[j / s]$. Otherwise, on a $\Psi$-based CSA, we start at $i=S A_{s}^{-1}[\lfloor j / s\rfloor]$ and the answer is $\Psi^{k}(i)$, for $k=j-\lfloor j / s\rfloor \cdot s$ (analogously 


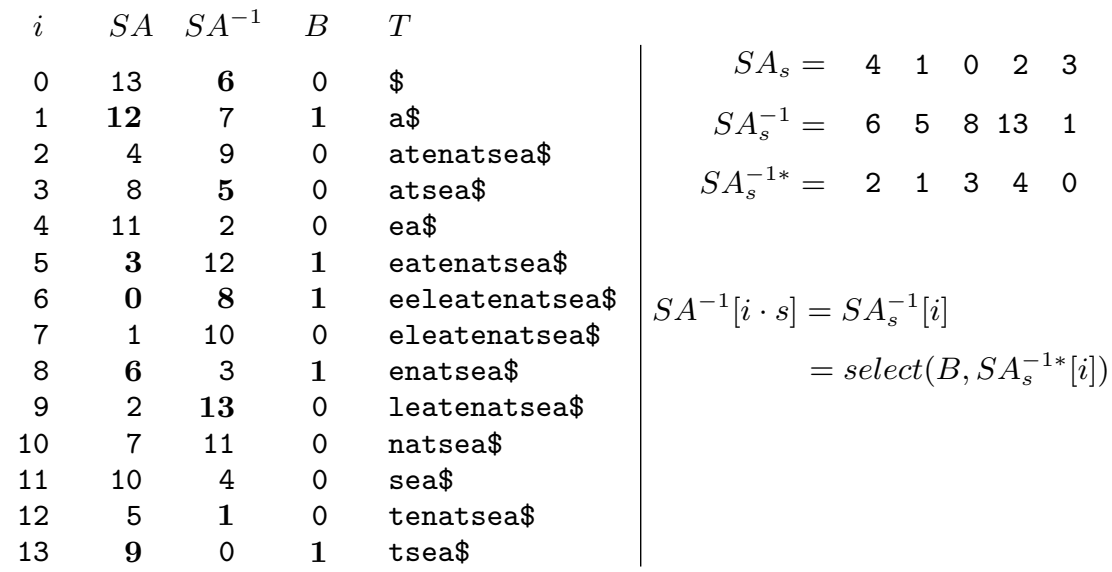

Fig. 1. Example of a suffix array, its inverse, and the sample arrays $S A_{s}$, the conceptional and formerly used $S A^{-1}$, and $S A^{-1 *}$ for $s=3$.

on an FM-index), taking up to $s$ steps. Computing $S A^{-1}[j]$ is useful in many scenarios, such as compressed suffix trees [3210] and document retrieval [33.

\section{A Combined Structure for Locating and Displaying}

In order to have a performance related to $s$ in locating and displaying text, the basic scheme uses $2(n / s) \lg n+O(n / s)+o(n)$ bits. In this section we show that this can be reduced to $(1+\epsilon)(n / s) \lg n+O(n / s)+o(n)$ bits, retaining the same locating cost and increasing the display cost to just $1 / \epsilon+s+r-l$ steps.

The key is to realize that $S A_{s}$ and $S A_{s}^{-1}$ are essentially inverse permutations of each other. Assume we store, instead of the value $i=S A_{s}^{-1}[j]$, the smaller value $i^{\prime}=S A_{s}^{-1 *}[j]=\operatorname{rank}_{1}(B, i)$. Since $B[i]=1$, we can retrieve $i$ from $i^{\prime}$ with the operation $i=\operatorname{select}_{1}\left(B, i^{\prime}\right)$, which finds the $i^{\prime}$ th 1 in $B$ and is implemented in constant time in the compressed representation of $B$ 28. Now, at the cost of computing select ${ }_{1}$ once when displaying a text range, we can store $S A_{s}^{-1 *}$ in $(n / s) \lg (n / s)+O(n / s)$ bits. What is more important, however, is that $S A_{s}$ and $S A_{s}^{-1 *}$ arrays are two permutations on $[0, n / s]$, and are inverses of each other. Fig. 1 shows an example.

Lemma 1. Permutations $S A_{s}$ and $S A_{s}^{-1 *}$ are inverses of each other.

Proof. $S A_{s}\left[S A_{s}^{-1 *}[j]\right]=S A_{s}\left[\operatorname{rank}_{1}\left(B, S A_{s}^{-1}[j]\right)\right]=S A\left[S A_{s}^{-1}[j]\right] / s=$ $S A\left[S A^{-1}[j \cdot s]\right] / s=(j \cdot s) / s=j$.

Munro et al. 23] showed how to store a permutation $\pi\left[0, n^{\prime}-1\right]$ in $(1+$ $\epsilon) n^{\prime} \lg n^{\prime}+O\left(n^{\prime}\right)$ bits, so that any $\pi(i)$ can be computed in constant time and any $\pi^{-1}(j)$ in time $O(1 / \epsilon)$. Basically, they add a data structure using $\epsilon n^{\prime} \lg n^{\prime}+O\left(n^{\prime}\right)$ bits on top of a plain array storing $\pi$. By applying this technique to $S A_{s}$, we retain the same fast access time to it, while obtaining $O(1 / \epsilon)$ time access to 
$S A_{s}^{-1 *}$ without the need to represent it directly. This yields the promised result (more precisely, the space is $(1+\epsilon)(n / s) \lg (n / s)+(n / s) \lg s+O(n / s)+o(n)$ bits). We choose to retain faster access to $S A_{s}$ because it is more frequently used, and for displaying the extra $O(1 / \epsilon)$ time cost is blurred by the remaining $O(s+r-l)$ time. One is free, of course, to choose the opposite.

Our experiments will show that this technique is practical and yields a significant improvement in the space-time tradeoff of CSAs, especially when the space is scarce and relatively large $s$ values must be used.

\section{A Structure for Prioritized Location of Occurrences}

Assume we want to locate only some, say $t$, occurrences of $P$ in $S A[s p, e p]$, as for example in many interfaces that show a few results. In a $\Psi$-based CSA, the number of steps needed to compute $S A[k]$ is its distance to the next multiple of $s$, that is, $D[k]=0$ if $S A[k]$ is a multiple of $s$ and $D[k]=s-(S A[k] \bmod s)$ otherwise. In an FM-index, the cost is $D[k]=S A[k] \bmod s$. We would like to use this information to choose low-cost entries $k \in[s p, e p]$ instead of arbitrary ones, as current CSAs do. The problem, of course, is that we do not yet know the value of $S A[k]$ before computing it! (some CSAs actually store the value $S A[k] \bmod s$ 29], but they are not space-competitive with the best current CSAs).

However, we do not need that much information. It is sufficient to know, given a range $D[x, y]$, which is the minimum value in that range. This is called a range minimum query $(\mathrm{RMQ})$ : $\mathrm{RMQ}(x, y)$ is the position of a minimum value in $D[x, y]$. The best current (and optimal) result for RMQs [9] preprocesses $D$ in linear time, producing a data structure that uses just $2 n+o(n)$ bits. Then the structure, without accessing $D$, can answer $\operatorname{RMQ}(x, y)$ queries in $O(1)$ time.

The basic method. We use the RMQ data structure to progressively obtain the values of $S A[s p, e p]$ from cheapest to most expensive, as follows [24]. We compute $k=\mathrm{RMQ}(s p, e p)$, which is the cheapest value in the range, retrieve and report $S A[k]$ as our first value, and compute $D[k]$ from it. The tuple $\langle s p, e p, k, D[k]\rangle$ is inserted as the first element in a min-priority queue that sorts the tuples by $D[k]$ values. Now we iteratively extract the first (cheapest) element from the queue, let it be the tuple $\langle l p, r p, k, v\rangle$, compute $k_{l}=\mathrm{RMQ}(l p, k-1)$ and $k_{r}=\mathrm{RMQ}(k+1, r p)$, then retrieve and report $S A\left[k_{l}\right]$ and $S A\left[k_{r}\right]$, and insert tuples $\left\langle l p, k-1, k_{l}, D[k]\right\rangle$ and $\left\langle k+1, r p, k_{r}, D[k]\right\rangle$ in the priority queue (unless they are empty intervals). We stop when we have extracted the desired number of answers or when the queue becomes empty. We carry out $O(t)$ steps to report $t$ occurrences 24 .

A stronger solution using $B$. Recall bitmap $B$ that marks the sampled positions. The places where $D[k]=0$ are precisely those where $B[k]=1$. We can use this to slightly reduce the space of the data structure. First, using operations rank $_{1}$ and select 1 on $B$, we spot all those $k \in[s p, e p]$ where $B[k]=1$. Only then we start reporting the next cheapest occurrences using the RMQ data structure as above. This structure, however, is built only on the entries of array 
$D^{\prime}$, which contains all $D[k] \neq 0$. Using rank $_{0}$ operations on $B$ (which counts $\left.0 \mathrm{~s}, \operatorname{rank}_{0}(B, i)=i-\operatorname{rank}_{1}(B, i)\right)$, we map positions in $D[l p, r p]$ to $D^{\prime}\left[l p^{\prime}, r p^{\prime}\right]$. Mapping back can be solved by using a select $t_{0}$ structure on $B$, but we opt for an alternative that is faster in practice and spends little extra memory: we create a sorted list of pairs $\left\langle k, \operatorname{rank}_{0}(B, k)\right\rangle$ for the already spotted $k$ with $B[k]=1$, and binary search it for mapping the positions back.

Refining priorities. The process can be further optimized by refining the ordering of the priority queue. Our method sorts the intervals [sp,ep] only according to the minimum possible value $u(=D[k])$. Assuming that the values in $D[s p, e p]$ are distributed uniformly at random in $[u, s)$ we can calculate the value of the expected minimum $\eta(l p, r p, u)=u+\sum_{v=u+1}^{s-1}\left(\frac{s-v}{s-u}\right)^{z}$, where $z=r p-l p+1$ is the range size. This can be used as a refined priority value.

Experiments. In the experimental section we will explore the performance of four solution variants: The 'standard' method, which extracts the first $t$ entries in $S A[s p, e p]$; a variant we call 'select', which enhances the baseline by using rank and select to first report all $S A[k]$ with $B[k]=1$; and the described RMQ approach on $D^{\prime}$, with the priority queue ordering according to the minimum value $D[k]$ ('RMQ') or the expected minimum in the intervals ('RMQ+est.min.').

Locating occurrences in text position order. By giving distinct semantics to the $D$ array, we can use the same RMQ-based mechanism to prioritize the extraction of the occurrences in different ways. An immediate application, already proposed in the literature (but not implemented) [26], is to report the occurrences in text position order, that is, using $D[k]=S A[k]$. In the experimental section we show that our implementation of this mechanism is faster than obtaining all the $S A[s p, e p]$ values and sorting them, even when a significant fraction of the occurrences is to be reported.

\section{Range-Restricted Location of Occurrences}

We now extend the mechanism of the previous section to address, partially, the more complex problem of retrieving the occurrences of $S A[s p, e p]$ that are within a text window $T[l, r]$. Again, we focus on retrieving some of those "valid" occurrences, not all of them. We cannot guarantee a worst-case complexity (as it would not be possible in succinct space [16]), but expect that in practice we perform faster than the baseline of scanning the values left to right and reporting those that are within the range, $l \leq S A[k] \leq r$, until reporting the desired number of occurrences.

If, as in the end of Section 4, we obtain the occurrences in increasing text position order, we will eventually report the leftmost occurrence within $T[l, r]$, and since then we will report all valid occurrences. As soon as we report the first occurrence position larger than $r$, we can stop. Although introducing ordering in the process, this mechanism is unlikely to be very fast, because it must traverse all the positions to the left of $l$ before reaching any valid occurrence. 
We propose the following heuristic modification, in order to arrive faster to the valid occurrences. We again store tuples $\langle l p, r p, k, S A[k]\rangle$, where $k$ gives the minimum position in $S A[l p, r p]$. But now we use a max-priority queue sorted according to $S A[k]$, that is, it will retrieve first the largest minima of the enqueued ranges. After inserting the first tuple as before, we iteratively extract tuples $\langle l p, r p, k, S A[k]\rangle$. If $S A[k]>r$, then the extracted range can be discarded and we continue. If $S A[k]<l$, then we split the interval into two as before and reinsert both halves in the queue (position $S A[k]$ is not reported). Finally, if $l \leq S A[k] \leq r$, we run the algorithm of the end of Section 4 on the interval $S A[l p, r p]$, which will give all valid positions to report. This process on $S A[l p, r p]$ finishes when we extract the first value larger than $r$, at which point this segment is discarded and we continue the process with the main priority queue.

Note that, although this heuristic is weaker in letting us know when we can stop, it is likely to reach valid values to report sooner than using the algorithm of Section 4. In the experimental section we will show that, although our technique is slower than the baseline for general intervals (e.g., near the middle of the text), it is faster when the desired interval is close to the beginning (or the end, as desired). This biased range-restricted searching is useful, for example, in versioned systems, where the latest versions are those most frequently queried.

\section{Experimental Results}

All experiments were run on a server equipped with $144 \mathrm{~GB}$ of RAM and two Intel Xeon E5640 processors each with a 12 MB L3 cache. We used the Pizza\&CHILI corpu $\$^{3}$ which contains texts from various application domains.

Our implementations are based on structures of version 2.0.2 of sDsL ${ }^{4}$ The CSAs of SDSL can be parameterized with the described traditional sampling method, which uses a bitmap $B$ to mark the sampled suffixes. It has recently been shown 11 that this sampling strategy, when $B$ is represented as sd_vector 27, gives better time/space tradeoffs than a strategy that does not use $B$ but samples every $S A[i]$ with $i \equiv 0 \bmod s$. In our first experiment, we compare the traditional sampling using $S A_{s}, S A_{s}^{-1}$ and $B$ to the new solution that replaces $S A_{s}^{-1}$ by just $\epsilon \cdot n / s$ samples plus a bitmap $B^{\prime}$ of length $n / s$ to mark those samples in $S A_{s}$. We opted for $\epsilon=1 / 8$, so that every $S A^{-1}$ value can be retrieved in at most 8 steps, and $B^{\prime}$ is represented as an uncompressed bitmap (bit_vector). The underlaying CSA is a very compact FM-index (csa_wt) parameterized with a Huffman-shaped wavelet tree (wt_huff) and a compressed bitmap (rrr_vector). We choose this FM-index deliberately, since the ratio of space assigned to samples is especially high. With the new method we save so much space that we can also afford to represent $B$ as bit_vector. Fig. 2 shows the time/space tradeoffs for accessing $S A$ and $S A^{-1}$ on one text (the results were similar on the others).

The points representing the same $s$ value lie further left in the new solution, since we saved space. Replacing $B$ with an uncompressed bitmap slightly reduces

\footnotetext{
3 Available under http://pizzachili.dcc.uchile.cl/.

${ }^{4}$ Publicly available under https://github.com/simongog/sdsl-lite
} 


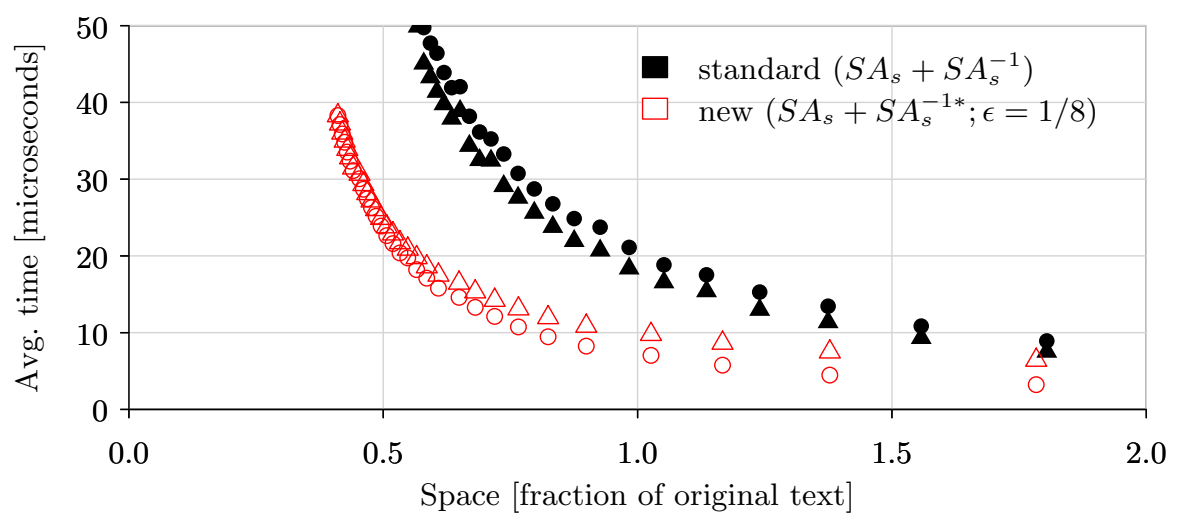

Fig. 2. Time/space tradeoffs to extract a $S A$ (o) respectively $S A^{-1}$ value $(\triangle)$ from indexes over input english.2108MB. Sampling density $s$ was varied between 1 and 32 .

access time, in addition. We only report this basic experiment, but we note that these better tradeoffs directly transfer to applications that need simultaneous access to $S A$ and $S A^{-1}$, like the child operation in compressed suffix trees.

In the second experiment, we measure the time to extract $t=50$ arbitrary $S A[k]$ values from a range $[s p, e p]$. We use the same FM-index of the previous paragraph. We create one index with $s=6$ and one using an RMQ structure on $D^{\prime}$ (rmq_succinct_sct). Setting $s^{\prime}=10$ for the latter index results in a size of $2,261 \mathrm{MB}$, slightly smaller than the $s=6$ index $(2,303 \mathrm{MB})$.

Fig. 3 (top) shows time and the average distance of the retrieved values to their nearest sample. Solution 'standard' spends the expected $\left(s^{\prime}-1\right) / 2=2.5 \mathrm{LF}$ steps per SA value, independently of the range size. Method 'select' first reports all sampled values in the range, hence the average distance linearly decreases and is close to zero at $s \times 50=300$. The RMQ based indexes spend the expected $(s-1) / 2=4.5$ steps for $z=50$. Using the RMQ information helps to decrease time and distance faster than linearly. The version using minimum estimation performs fewer LF steps for ranges in [150,220], but the cost of the RMQs in this case is too high compared to the saved LF steps.

In scenarios where LF is more expensive, 'RMQ+est.min.' can also outperform 'select' in runtime. The cost of one LF step depends logarithmically on the alphabet size $\sigma$, while the RMQ cost stays the same. Thus, using a text over a larger alphabet yields a stronger correlation between the distance and runtime, as shown in Fig. 3 (bottom), where we repeat the experiment using an FM-index (csa_wt parameterized with wt_int) on a word parsing enwiki.4646MB of the English Wikipedia ( $\sigma=3,903,703)$. The RMQ supported index takes $3362 \mathrm{MB}$ for $s^{\prime}=10$ and we get $3393 \mathrm{MB}$ for $s=6$.

Using almost the same index on english.2108MB (RMQ is built on $S A$ this time, using $s^{\prime}=32$ and $s=10$, obtaining sizes 1,554 MB and 1,590 MB), we now evaluate how long it takes to report the $t=10$ smallest $S A[k]$ values in a range $S A[s p, e p]$. The standard version sequentially extracts all values in 

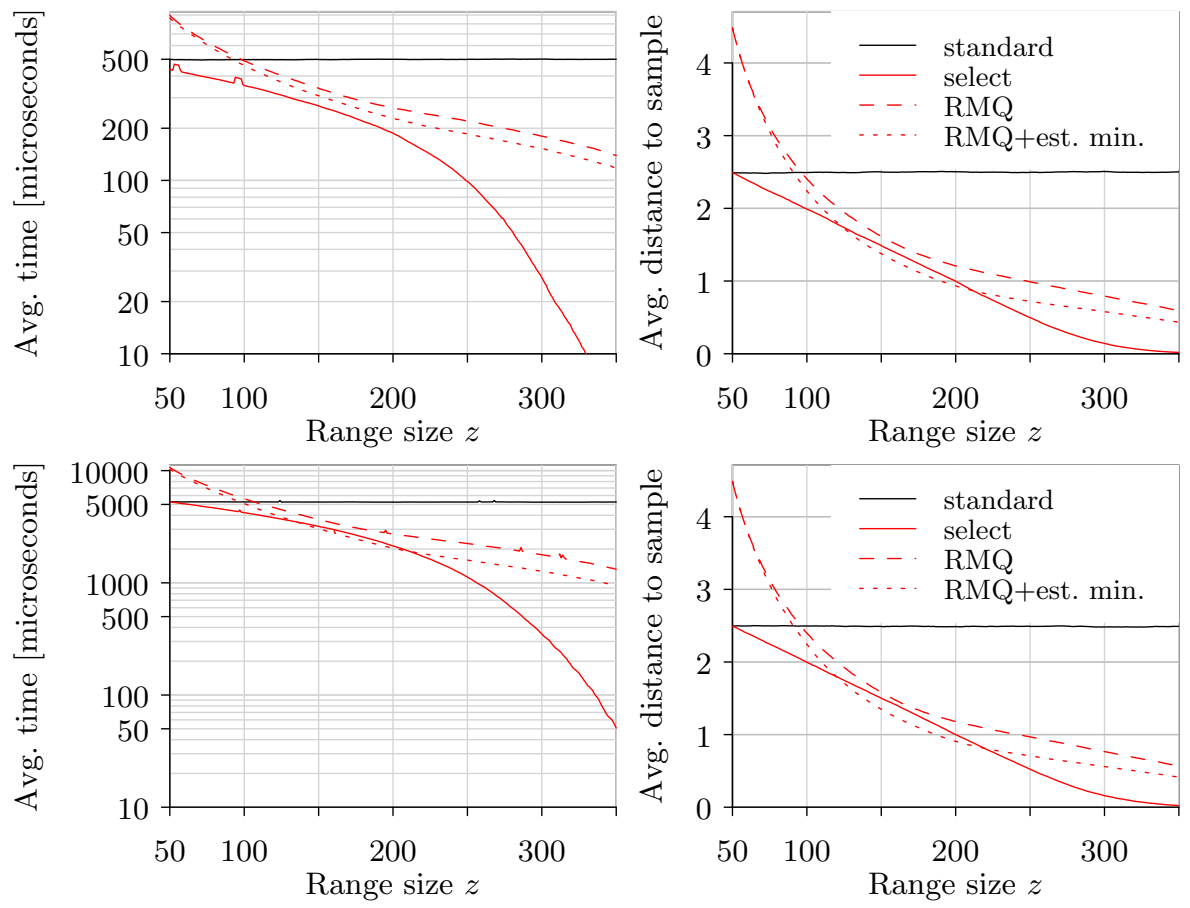

Fig. 3. Left: Time to report 50 values in the range $S A[s p, s p+z-1]$. Right: Distance of a reported $S A[k]$ value to its nearest sample. Input: english.2108MB (top) and enwiki.4646MB (bottom).

$S A[s p, e p]$, while keeping a max-priority queue of size $k$ with the minima. The RMQ based method uses a min-priority queue that is populated with ranges and corresponding minimum values. Fig. 4 contains the results. For range size $z=10$, the standard method is about 3 times faster, since we decode 10 values in both methods and the sampling of the standard method is 3.2 times denser than that of the RMQ supported index. The RMQ index extracts $2 t-1$ values in the worst case, when there are $t-1$ left in the priority queue. Therefore it is not surprising that the crossing point lies at about $60 \approx 3.2 \times(2 t-1)$.

Lastly, we explore the performance of range-restricted locating on the same indexes. We take pattern ranges of size 10,000 and search for occurrences in text ranges $T[l, l+0.01 n]$, which corresponds to the scenario drawn earlier in the paper. Fig. 5 shows that our heuristic using a max-priority queue to retrieve subranges that contain values $\geq l$, is superior to the standard approach in the first $15 \%$ of the text. The approach of the previous experiment extracts first all the occurrences located in $T[0, l-1)$, and thus becomes quickly slower than the standard approach. 


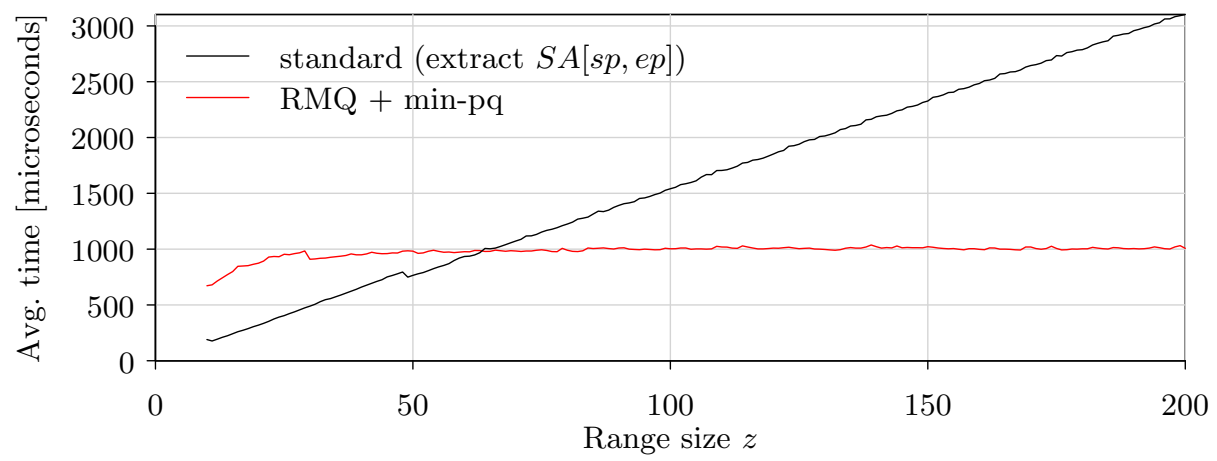

Fig. 4. Average time to report the ten smallest $S A[k]$ values in $S A[s p, s p+z-1]$.

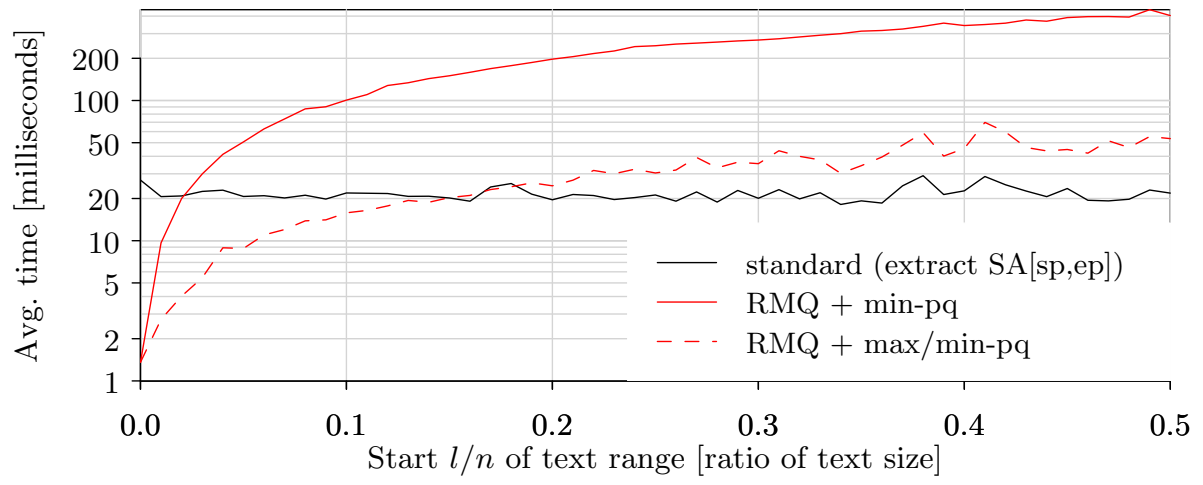

Fig. 5. Average time to report ten $S A[k] \in[l, r]$ for $k$ in $[s p, s p+10000-1]$.

\section{References}

1. D. Belazzougui and G. Navarro. Alphabet-independent compressed text indexing. In Proc. ESA, pages 748-759, 2011.

2. P. Bille and I. Gørtz. Substring range reporting. In Proc. CPM, LNCS 6661, 2011.

3. M. Burrows and D. Wheeler. A block sorting lossless data compression algorithm. Technical Report 124, Digital Equipment Corporation, 1994.

4. P. Ferragina, R. González, G. Navarro, and R. Venturini. Compressed text indexes: From theory to practice. ACM J. Exp. Alg., 13:art. 12, 2009.

5. P. Ferragina and G. Manzini. Opportunistic data structures with applications. In Proc. FOCS, pages 390-398, 2000.

6. P. Ferragina and G. Manzini. Indexing compressed texts. J. ACM, 52(4):552-581, 2005.

7. P. Ferragina, G. Manzini, V. Mäkinen, and G. Navarro. Compressed representations of sequences and full-text indexes. ACM Trans. Alg., 3(2):article 20, 2007.

8. P. Ferragina, J. Sirén, and R. Venturini. Distribution-aware compressed full-text indexes. Algorithmica, 67(4):529-546, 2013.

9. J. Fischer and V. Heun. Space-efficient preprocessing schemes for range minimum queries on static arrays. SIAM J. Comp., 40(2):465-492, 2011.

10. J. Fischer, V. Mäkinen, and G. Navarro. Faster entropy-bounded compressed suffix trees. Theor. Comp. Sci., 410(51):5354-5364, 2009. 
11. Simon Gog and Matthias Petri. Optimized succinct data structures for massive data. Soft. Prac. \& Exp., 2013. http://dx.doi.org/10.1002/spe.2198, (to appear).

12. G. Gonnet, R. Baeza-Yates, and T. Snider. Information Retrieval: Data Structures and Algorithms, chapter 3: New indices for text: Pat trees and Pat arrays, pages 66-82. Prentice-Hall, 1992.

13. R. Grossi, A. Gupta, and J. S. Vitter. High-order entropy-compressed text indexes. In Proc. SODA, pages 636-645, 2003.

14. R. Grossi and J. Vitter. Compressed suffix arrays and suffix trees with applications to text indexing and string matching. In Proc. STOC, pages 397-406, 2000.

15. R. Grossi and J. Vitter. Compressed suffix arrays and suffix trees with applications to text indexing and string matching. SIAM J. Comp., 35(2):378-407, 2006.

16. W.-K. Hon, R. Shah, S. . Thankachan, and J. Vitter. On position restricted substring searching in succinct space. J. Discr. Alg., 17:109-114, 2012.

17. J. Kärkkäinen, P. Sanders, and S. Burkhardt. Linear work suffix array construction. J. ACM, 53(6):918-936, 2006.

18. D. Kim, J. Sim, H. Park, and K. Park. Constructing suffix arrays in linear time. J. Discr. Alg., 3(2-4):126-142, 2005.

19. P. Ko and S. Aluru. Space efficient linear time construction of suffix arrays. $J$. Discr. Alg., 3(2-4):143-156, 2005.

20. V. Mäkinen and G. Navarro. Rank and select revisited and extended. Theor. Comp. Sci., 387(3):332-347, 2007.

21. U. Manber and G. Myers. Suffix arrays: a new method for on-line string searches. SIAM J. Comp., 22(5):935-948, 1993.

22. G. Manzini. An analysis of the Burrows-Wheeler transform. J. ACM, 48(3):407430, 2001.

23. J. Munro, R. Raman, V. Raman, and S. Rao. Succinct representations of permutations. In Proc. ICALP, LNCS n. 2719, pages 345-356, 2003.

24. S. Muthukrishnan. Efficient algorithms for document retrieval problems. In Proc. SODA, pages 657-666, 2002.

25. G. Navarro and V. Mäkinen. Compressed full-text indexes. ACM Comp. Surv., 39(1):art. 2, 2007.

26. Y. Nekrich and G. Navarro. Sorted range reporting. In Proc. SWAT, LNCS 7357, pages 271-282, 2012.

27. D. Okanohara and K. Sadakane. Practical entropy-compressed rank/select dictionary. In Proceedings of the Workshop on Algorithm Engineering and Experiments. SIAM, 2007.

28. R. Raman, V. Raman, and S.S. Rao. Succinct indexable dictionaries with applications to encoding $k$-ary trees, prefix sums and multisets. ACM Trans. Alg., 3(4):art. 43, 2007.

29. S. Rao. Time-space trade-offs for compressed suffix arrays. Inf. Proc. Lett., 82(6):307-311, 2002.

30. K. Sadakane. Compressed text databases with efficient query algorithms based on the compressed suffix array. In Proc. ISAAC, LNCS v. 1969, pages 410-421, 2000.

31. K. Sadakane. New text indexing functionalities of the compressed suffix arrays. $J$. Alg., 48(2):294-313, 2003.

32. K. Sadakane. Compressed suffix trees with full functionality. Theor. Comp. Sys., 41(4):589-607, 2007.

33. K. Sadakane. Succinct data structures for flexible text retrieval systems. J. Disc. Alg., 5(1):12-22, 2007.

34. P. Weiner. Linear pattern matching algorithm. In Proc. 14th IEEE Symposium on Switching and Automata Theory, pages 1-11, 1973. 